\title{
Probiotic and Prebiotic Applications in Aquaculture
}

\section{Daniel Merrifield ${ }^{1 *}$ and Zhigang Zhou ${ }^{2 *}$}

${ }^{1}$ Aquatic Animal Nutrition and Health Research Group, School of Biomedical and Biological Sciences, Plymouth University, Plymouth, UK

${ }^{2}$ Department of Aqua Feed and Nutrition, Feed Research Institute, Chinese Academy of Agricultural Sciences, Beijing, China

In this special issue, the journal publishes articles on gut microbiology, probiotics and prebiotics in the field of aquaculture. Probiotics and prebiotics have emerged as exciting feed additives and over the past 10-15 years a great deal of research has highlighted the potential benefits for aquatic animal health and performance. However, we are very much at a transitional stage, moving beyond proof of concept and into practical applications at the industrial level. Indeed, probiotics and prebiotics are now routinely incorporated into commercial aquafeed formulations and within the short to medium term we will be better placed to understand the practical and economic benefits of these applications.

Although we already have a broad knowledge base with regards to the effect on host innate immunity at the systemic level, our understanding of the important host-microbe interactions at the mucosal interface and the subsequent localised immunological responses is lacking. However, during the last few years a number of papers have revealed important information on the localised host response to gut microbes and probiotics with respect to the gene expression of pro- and anti-inflammatory cytokines (e.g. IL$1 \beta$, IL-8, IL-10 and TNFa), mucosal antibodies (i.e. IgT/IgZ), TLR's, various other important immunological proteins and proteins involved in the regulation of cellular activity and apoptosis (e.g. PCNA and Hsp70).

In order to further our relatively limited understanding we must follow the blueprint of mammalian research and employ proteomic techniques in conjunction with metagenomics. The utilisation of gnotobiotic systems in this context, which have begun to emerge with live feeds, European sea bass, Atlantic cod and zebrafish, should provide a solid understanding of the true extent of the influence of gut microbes on fish biological functions, with particular emphasis on co-metabolism, gastric development and immunity.

As this information emerges we will be better placed to make informed decisions on how to employ probiotics and prebiotics in order to achieve optimal benefits.

The editors and publishers are grateful for the thorough contributions of the review panel:

\section{Reviewer panel}

\begin{tabular}{|l|l|}
\hline A. Abid, UK & J. Pintado, Spain \\
\hline L. Alfonso, Canada & T. Perez-Sanchez, Spain \\
\hline M. Avella, USA & M.D. Rawling, UK \\
\hline J.L. Balcazar, Spain & A. Ray, India \\
\hline A. Dimitroglou, Greece & E. Ringø, Norway \\
\hline D.Guroy, Turkey & A. Rodiles, Spain \\
\hline S. Hoseinifar, Iran & J. Romero, Chile \\
\hline M. Kiernan, UK & A. Shenavar, Iran \\
\hline H. Kuehlwein, UK & B. Standen, UK \\
\hline M.R. Liles, USA & Y.Z. Sun, China \\
\hline P. Makridis, Greece & P. Waines, UK \\
\hline P. Navarrete, Chile & L. Weifen, China \\
\hline R. Opazo, Chile & \\
\hline
\end{tabular}

*Corresponding authors: Dr. Daniel Merrifield,Aquatic Animal Nutrition and Health Research Group, School of Biomedical and Biological Sciences, Plymouth University, Plymouth, UK, E-mail: daniel.merrifield@plymouth.ac.uk

Dr. Zhigang Zhou, Department of Aqua Feed \& Nutrition, Feed Research Institute, Chinese Academy of Agricultural Sciences, Beijing, China, E-mail: zhou_zg@msn. com

Received November 09, 2011; Accepted November 10, 2011; Published November 14, 2011

Citation: Merrifield D, Zhou Z (2011) Probiotic and Prebiotic Applications in Aquaculture. J Aquac Res Development S1:e001. doi:10.4172/2155-9546.S1-e001

Copyright: (c) 2011 Merrifield D, et al. This is an open-access article distributed under the terms of the Creative Commons Attribution License, which permits unrestricted use, distribution, and reproduction in any medium, provided the original author and source are credited. 